\title{
A Rare Case of Acute Onset of Hydrocephalus in a Nineteen-Year-Old Patient
}

\author{
Hasan Nasir ${ }^{1}$, Darren Nguyen ${ }^{1}$, Hamza Sultan ${ }^{1}$, Nalin Ranasinghe ${ }^{2}$ and Leonard Ranasinghe ${ }^{3 *}$ \\ ${ }^{1}$ First year medical students, California Northstate University College of Medicine, USA \\ ${ }^{2}$ Director of Emergency Medicine, AO Fox Hospital, Oneonta, NY. Assistant Clinical Professor, California Northstate University College of Medicine, USA \\ ${ }^{3}$ Professor of Emergency Medicine and Clerkship Director of Emergency Medicine, California Northstate University College of Medicine, USA
}

*Corresponding author: Leonard Ranasinghe, MD, California Northstate University College of Medicine, 9700 W. Taron Dr., Elk Grove, California, 95757, USA.

To Cite This Article: Hasan Nasir, Darren Nguyen, Hamza Sultan, Nalin Ranasinghe, Leonard Ranasinghe. A Rare Case of Acute Onset of Hydrocephalus in a Nineteen-Year-Old Patient. AmJ Biomed Sci \& Res. 2021 - 13(4). AJBSR.MS.ID.001892. DOI: 10.34297/AJBSR.2021.13.001892.

Received: 粗 June 18, 2021; Published: 眥 July 19, 2021

\begin{abstract}
Hydrocephalus is a rare disorder characterized by the accumulation of cerebrospinal fluid (CSF) inside the ventricles of the brain. The disease has multiple etiologies including, but not limited to, congenital malformations, neoplasms, and infections. The case discussed in this report pertains to a 19-year-old male who presented with acute hydrocephalus. Initial workups such as physical exams, CBC, chemistry panel, CT imaging and ECG were performed. Furthermore, initial treatment with Diamox and dexamethasone was started. The patient was then moved to the neurosurgery department at a tertiary hospital where an EVD was placed. Unfortunately, the patient had a complication during the EVD placement and developed an epidural hematoma and swelling in the posterior fossa a few days afterward. Furthermore, the patient was diagnosed with a Factor VII deficiency and started on replacement therapy. Despite medical treatment, the patient's condition continued to deteriorate, and he passed away 44 days after admission into the tertiary hospital. Due to the patient's age group as well as the presence of a concurrent clotting disorder, ascertaining the ideal treatment proved to be difficult. Therefore, a review of the case as well as meta-analysis of related articles may shine a light on how to not only diagnose the underlying causes of hydrocephalus but also discuss strategies that can be undertaken if comorbid conditions are present.
\end{abstract}

Keywords: Hydrocephalus; Cerebral aqueduct stenosis; External ventricular drain; Factor VII deficiency

Abbreviations: CSF: Cerebrospinal Fluid; EVD: External Ventricular Drain; VP: Ventriculoperitoneal; PERRLA: Pupils Equal Round Reactive (to) Light Accommodation; DNR: Do Not Resuscitate

\section{Introduction}

The goal of this case report is to examine the atypical case of acute hydrocephalus in the patient. Using prior literature on the condition's pathogenesis, we aim to examine significant clinical findings which would guide prompt diagnosis and management of this debilitating condition. In most cases, management is precarious, so it is important to discuss the different strategies used in treating patients with hydrocephalus to enhance informed decisionmaking by physicians. Ultimately, the case report contributes to the limited pool of information regarding acute hydrocephalus in the adolescent population.

Hydrocephalus is broadly defined as a build-up of a significant amount of CSF in the intracerebral ventricles of the brain. The typical patient is either an older adult or an infant presenting with variable pathologies attributable to weakened structural support of the ventricular system [1]. The worldwide prevalence for adults (19-64-year-old) diagnosed with acute hydrocephalus is $0.003 \%$ [2]. Multiple pathologies may be responsible for an acute or chronic onset of this condition. Acute hydrocephalus is typically an idiopathic condition, usually occurring after a subarachnoid hemorrhage, pineal tumor, or post-infection. Commonly, the pathogenesis occurs due to an obstruction of the flow of CSF between ventricles [3]. Secondary neurological complications can occur due to brain compression aggravated by increased intracranial pressure. Some prominent neurological signs in young adults include sudden-onset headache, blurred vision, papilledema, 
and unstable balance [4]. The broad nature of the symptoms may make the diagnosis difficult to ascertain in a patient, especially one who deviates from the typical demographic. Immediate diagnosis and treatment of hydrocephalus is necessary to reduce the onset of permanent disability and improve patient outcomes [4].

Several studies have been conducted to address the surgical management of hydrocephalus. One case series study observed instances of hydrocephalus specifically involving masses in the lateral ventricle. The study consisted of 11 cases of lateral ventricular lesions with 9 of the 11 cases presenting with preoperative hydrocephalus. All the participants had an atypical age of presentation with the mean age being 25 years old ranging from 6 to 45 years of age. The most common pathological lesion, resulting in hydrocephalus, was a neurocytoma observed in $27.3 \%$ of cases. This was followed by grade 1 meningioma making up $18.2 \%$ of cases. Although total resection was performed in all cases, four patients developed severe complications such as intraventricular bleeding and ventriculitis. Only two patients did not require an external ventricular drain (EVD) postoperatively to relieve the hydrocephalus with both being admitted for a grade 1 meningioma. Two patients developed iatrogenic hydrocephalus because of the procedure with one patient requiring a ventricular shunt to relieve the symptoms. This study demonstrates the stringent precautions that must be taken in managing hydrocephalus through resections. Complications can readily occur, and these procedures may not necessarily provide a safeguard against future instances of hydrocephalus [5].

Congenital mutations can also result in hydrocephalus. Although most of these defects present early, in infancy, there are instances of where hydrocephalus develops later in life. One publication looked at 25 cases of late onset hydrocephalus with the age of the patients ranging from 5 to 64 years. All the patients had a diagnosis of aqueduct stenosis. The most common age group with the condition in this study was 40-64 years followed by 5-20 years. The study had a predilection toward males with them making up $72 \%$ of the cases. In $80 \%$ of cases, a VP shunt resulted in marked improvement with the most common complication being subdural hematoma or post-surgical infection [6].

Furthermore, some studies have been done to examine the long-term effects of hydrocephalus in adolescents and children. Another study analyzed the effects of shunt treatment for 138 patients of ages 15 years and younger who had hydrocephalus. The study found that the mortality rate was $21.7 \%$ with no differences in rate between the first and second decade. Furthermore, $81 \%$ of patients needed their shunt revised while $10 \%$ did not. In terms of employment, $56 \%$ of all patients were either students or working while $21 \%$ were unemployed. Lastly, while the health-related quality of life scores are lower than the general population, most patients reported living lives that are "comparable" to their friends and family [7]. The long term neurological or cognitive damages caused by hydrocephalus are worth noting especially for young patients. Detrimental lifelong neurological or cognitive effects may occur and can significantly impact career and lifestyle options.

Despite the inherent risk of surgical intervention in treating hydrocephalus, comorbidities may make treatment much more complicated. A dual case study looked at two infants with hydrocephalus concurrent with hemophilia A. The key decision that proved to be successful in treating both these patients was that their factor VIII deficiency was diagnosed before any surgical intervention was made. Because of this diagnosis, both infants were started on preoperative factor VIII replacement therapy for at least a week before surgery. The first case was given an EVD later replaced by a VP shunt while the second case presented with a Dandy Walker malformation and was given a VP shunt. The patients in both cases demonstrated remarkable recovery and were on track for hitting developmental milestones in a follow up appointment. Although hemophilia A can present much more severely than a factor VII deficiency, the general principles of preoperative management remain the same for treating hydrocephalus in patients with a comorbid clotting disorder [8]. A review of the following case should demonstrate how to diagnose and important considerations that need to be taken in treating hydrocephalus concurrent with a clotting disorder.

\section{Methods}

The information pertinent to the case was relayed to the research team by the site advisor who was involved in the treatment of the patient in the Emergency Department. Information was deidentified and deemed HIPAA-compliant prior to distribution to the research team for use in the development of the case report. After receiving the information, research questions were formed which guided the literature review. The first goal was to ascertain the rarity of the disease given the patient's age. The second goal was to analyze the pathology of acute hydrocephalus and apply it to the patient's presentation in the emergency department. The third goal was to use relevant literature to determine management strategies that may be used in the patient's case.

To understand the pathology of acute hydrocephalus, a literature review was conducted using the following medical databases: National Center for Biotechnology Information (NCBI); PLOS One; and Access Medicine. The following keywords were used in the search of pertinent information: Acute Hydrocephalus; young; prognosis; diagnosis; treatment; symptoms. After discussing the literature, the information most pertinent to the case was used to develop the Introduction and Discussion Section. The sections were discussed at great length for peer revision to ensure quality standards were met in the creation of the case report sections. 


\section{Discussion}

The patient was a 19-year-old male with a history of depression and drug use who presented to the emergency room with dizziness along with unfocused vision for the last 2-3 weeks. While waiting in the hospital, the patient suffered an acute episode of syncope resulting in facial bruising and neck pain with the extent of his head injuries being unclear. The patient denied nausea, vomiting, or headache associated with his dizziness. He had also not experienced any dyspnea, paresthesia, chest pain or abdominal pain. Furthermore, he did not have a loss of limb, bowel, or bladder function. Lastly, the patient denied thoughts of self-harm, or any hallucinations. Other than the neurological symptoms the patient reported, he stated that he contracted tetanus in 2018. Additional information on the patient's past medical history, family history, and social history was not declassified for use in this study so no definitive analysis can be made regarding these factors.

On physical examination, the patient was alert and oriented. $\mathrm{He}$ was afebrile at 36.7 degrees Celsius. He had a stable heart rate of $76 \mathrm{bpm}$, a respiratory rate of 18 breaths per minute, and an oxygen saturation of $96 \%$. His blood pressure was measured at $139 / 68$ $\mathrm{mm} \mathrm{Hg}$ indicating an elevated pulse pressure. He had no difficulty speaking, breathing, and displayed good strength with normal sensations. The patient exhibited PERRLA on the eye examination. His lungs were clear to auscultation, and the patient had no abdominal tenderness. Outside of the tenderness in the periorbital area with bruising, he was normocephalic and atraumatic. On examination, there was no spinal tenderness, but his neck did have discomfort.

As part of the workup for the patient, a CBC with differential, chemistry panel, CT of the cervical area and ECG were ordered. The CBC test revealed leukocytosis at 13 cells/uL with $80.6 \%$ neutrophil and $10.3 \%$ lymphocyte relative count. Furthermore, the patient was found to have thrombocytosis at $555 \times 103$ cells/uL. The chemistry panel also detected some abnormalities regarding the patient. Calcium and total bilirubin level were elevated at $11.1 \mathrm{mg} /$ $\mathrm{dL}$ and $1.7 \mathrm{mg} / \mathrm{dL}$ respectively. Looking further into the bilirubin count, the patient was found to have an elevated direct bilirubin level of $0.4 \mathrm{mg} / \mathrm{dL}$ and an elevated indirect bilirubin count of $1.3 \mathrm{mg} /$ dL. Lastly, the patient had a D-Dimer level less than $215 \mathrm{ng} / \mathrm{mL}$ and a low Creatine Phosphokinase level at $57 \mathrm{U} / \mathrm{L}$. To investigate potential neurological etiologies of the patient's condition, a noncontrast CT scan was done in three areas of the patient: the brain, face, and cervical spine. The CT scans of the head and cervical spine are indicated by images A and B respectively. Imaging of the brain revealed dilation of lateral ventricles, 3 rd ventricle and the cerebral aqueduct. The 4 th ventricle, however, was normal in size. The presence of this abnormality definitively indicates the presence of obstructive hydrocephalus. Furthermore, hypoattenuation was detected around the ventricles in the cerebral and cerebellar area. The CT scan found no extra-axial fluid accumulation or depressed skull fractures. Next, facial area imaging found no signs of fractures, temporomandibular joint dislocation, orbital abnormalities, or sinus obstructions. Lastly, while CT imaging of the cervical spine indicated misalignment of the $\mathrm{C} 5$ and $\mathrm{C} 6$ vertebral bodies, this was likely attributed to motion artifact. In addition, other significant findings include disc narrowing between $\mathrm{C} 7$ and T1 and a hypodense appearance of the cervical spinal cord. Other than the abnormal findings, imaging indicated proper cervical spine alignment with no fractures, or prevertebral edema.

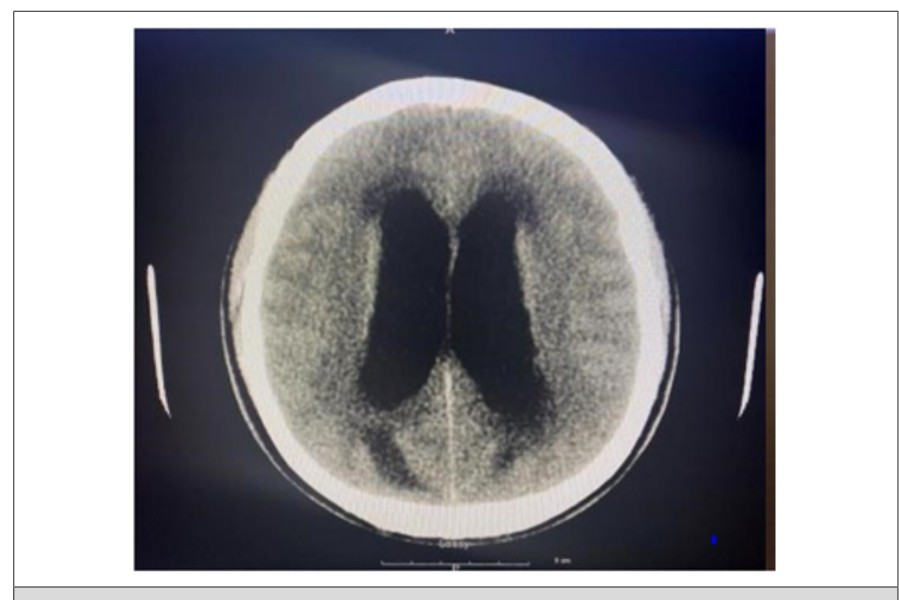

Figure A: Head CT.

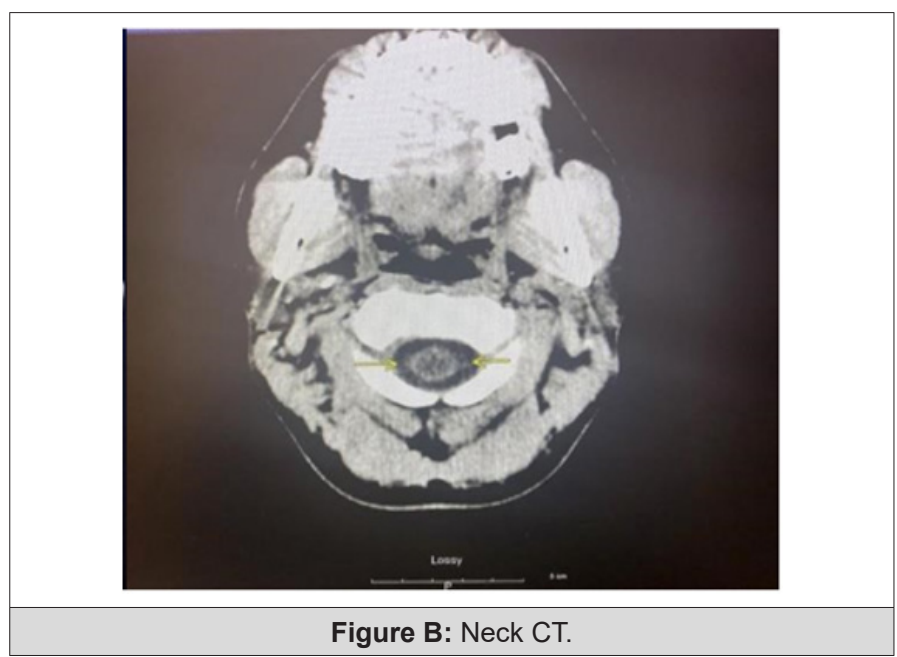

Next, the patient was prescribed intravenous fluids, acetaminophen, bacitracin ointment for the bruising. In addition, 1 gram of acetazolamide, and 10 milligrams of dexamethasone were prescribed. Eventually, the patient was transferred to neurosurgical care and then transferred out of the initial hospital to a tertiary hospital for more intensive treatment. The neurosurgery team at the new hospital evaluated the patient by an MRI which was given with and without contrast. The images displayed non-communicating hydrocephalus with obstruction at the cervicomedullary junction 
along with diffuse ventriculitis. The patient also had cerebellar edema, and hydromyelia. The images also exhibited enhancement of his ependymal lining from the cervicomedullary junction to approximately T9. The images were not shared with the patient information, so they have not been included in this report. On the second day of his admission, the patient decompensated resulting in the placement of an EVD.

After the procedure, a novel epidural hematoma formed. The patient was then intubated and taken to the OR for an emergent craniotomy along with a hematoma evacuation. On the 6th day of hospitalization, the patient was re-intubated to manage respiratory distress. The next day, the neurologic status of the patient worsened, requiring immediate suboccipital craniectomy for the posterior fossa decompression, and resection of the fourth ventricular cystic dilation. Laboratory studies discovered factor VII deficiency, and the patient was soon placed on factor VII replacement therapy to minimize risk of severe bleeding.

More procedures were ordered to improve the patient's outcomes. 19 days after admission, a percutaneous endoscopic gastrostomy tube placement and a tracheostomy were performed on the patient. About 2 weeks later, the patient was sent to the Operating room for a septostomy to treat persistent hydrocephalus. A week after the procedure, a DNR was signed by the patient and comfort measures were taken. Two days later, the patient passed away: 44 days after admission to the tertiary hospital. Based upon risk assessment determined by the typical demographics diagnosed with acute hydrocephalus, it is very rare for a 19-yearold to present with this condition. As mentioned earlier, the typical patients of hydrocephalus are either infants or the elderly. Given the low rate of prevalence as well as his young age, the chief complaint of the patient may not immediately bring about the diagnosis of acute hydrocephalus, as conditions such as internal bleeding and heart issues are more common causes of the patient's symptoms according to the patient's demographic [3]. Nonetheless, previous literature related to management of hydrocephalus indicates that without proper inspection, improper diagnosis and treatment of the condition may lead to severe, irreversible damage [4].

In terms of prognosis for the patient, studies show that significant deleterious effects may occur in the long run if hydrocephalus is not treated promptly [5]. Therefore, it is imperative to find the underlying etiology that led to the occurrence of hydrocephalus within the patient. The most probable cause of noncommunicating hydrocephalus within the patient's age demographic is a ventricular lesion [5]. Nevertheless, no specific tumors were identified on the CT or the MRI making the presence of an obstructive neoplasm unlikely. A potential differential diagnosis can be made for idiopathic cerebral aqueduct stenosis based on the CT imaging indicating dilation of the first three ventricles but not the fourth cerebral ventricle.
Other differentials can also be explored based on the initial imaging of the patient. For instance, the early radiologic finding of ependymal lining enhancement, extensive to the level of T9, is highly suspect of ventriculitis. However, multiple pathologies could explain this event. Although a shunt may be able to explain this finding, leakage of the CSF, as well as meningitis are other possible causes [3]. While the discovery preceded surgical intervention in this patient, ventriculitis may also occur in response to invasive surgical procedures which manage hydrocephalus [4]. Additional analysis of the CT performed on the head, and cervical spine demonstrates changes in neuroanatomy, such as dilated ventricles, resulting from high intracranial pressure. These findings can additionally point towards potential causes of the hydrocephalus. For example, in noncommunicating hydrocephalus caused by spinal cord edema, swelling that extends to at least two vertebral segments above an injured portion is a diagnostic finding [9]. Furthermore, the absence of extra-axial fluid collection is used to rule out potential hydrocephalus ex-vacuo [10].

In the case of the patient, hydrocephalus continued following the placement of an external ventricular drain. The persistence of the hydrocephalus suggests either unmanageable severity of the extent of the hydrocephalus, or complications related to the Factor VII deficiency. The low D-dimer on the patient's CBC is probably indicative of the Factor VII deficiency which he was diagnosed with a week after admission. The most common manifestations of this condition are easy bruising, gum and nose bleeds [11]. Life threatening complications are rare but usually involve bleeds in the central nervous or gastrointestinal systems [11]. While it is unknown how severe the patient's deficiency was, it can be safe to say that invasive operations in the brain would only increase his risk of hemorrhage [12].

The decision of whether or not to place a ventricular shunt depends on the assessment by the neurological care team.13 A ventricular shunt is contraindicated if there is active infection of the CSF or the obstruction area, coagulopathy, high CSF protein, and blood in CSF [12]. The most significant complications associated with surgical treatment of hydrocephalus is hemorrhage [13]. EVD's are associated with lower rates of hemorrhage as opposed to VP shunts with the most prevalent risk factor being the use of perioperative antiplatelet medications [13]. Although it is unknown what medications the patient was given by the neurosurgical team, the patient's undiagnosed Factor VII deficiency may have resulted in an increased risk of bleeding during the procedure [12]. It is likely that the epidural hematoma was a complication of the operation. Since the only treatment of an epidural hematoma is surgery, the patient may have been at an even greater risk of further hemorrhage due to the craniotomy. This risk is evidenced by the fact that within the next week, the patient had to undergo a craniectomy due to compression of the posterior fossa due to severe dilation 
of the fourth ventricle. Despite the EVD being placed, it was not particularly efficacious in treating the hydrocephalus. It was likely that the continued hemorrhages due to the procedures continued to cause swelling within the brain. The management of hydrocephalus with a comorbid clotting disorder makes the treatment much more complicated. In similar cases with a more favorable outcome, the key difference was that their clotting disorder was diagnosed early, and they were treated promptly before any surgical procedures were undertaken [8]. The patient on the other hand was operated on multiple times before the diagnosis of Factor VII deficiency was made. If this diagnosis was made earlier, then the patient may have had a more favorable outcome.

As mentioned in the introduction, while alterations and future procedures may be necessary to manage atypical hydrocephalus, the patient may have led a relatively normal life if his condition was promptly managed [5]. The patient's age may have been a contributing factor to why the diagnosis of Factor VII deficiency was made so late. Typically, the symptoms of the disorder should have become prevalent early during the patient's life. The neurosurgical team could have simply not considered this disorder preoperatively since it would have been noted in the patient's history at his age. Due to the patient's incomplete history, one can only speculate on why the diagnosis was not made during childhood. The most likely cause is that the patient simply did not have proper access to healthcare resources. It is important to consider that young patients presenting with hydrocephalus should always have a workup done for a clotting disorder.

\section{Conclusion}

The presence of hydrocephalus within the patient is uncharacteristic due to his age. Although the presence of a ventricular lesion is most likely, there were no obstructive masses seen on imaging. The elevated intracranial pressure is likely due to a cerebral aqueduct stenosis judging from the pattern seen on the CT. The treatment of the patient was complicated by the presence of an underlying Factor VII deficiency. If this disorder were diagnosed earlier, proper measures could have been taken to prevent perioperative hemorrhage. The late onset of symptoms makes this patient's case highly peculiar as there is little to no literature discussing what factors can cause such a delayed presentation of congenital hydrocephalus. With this fact, other diagnoses should still be considered, but with the lack of a patient history, it is difficult to consider all the factors.

\section{Conflicts of Interests}

No conflicts of interest are indicated in this case report.

\section{References}

1. Rekate H L (2008) The definition and classification of hydrocephalus: a personal recommendation to stimulate debate. Cerebrospinal Fluid Res $5(2)$.

2. Isaacs A M, Riva Cambrin J, Yavin D, Hockley A, Pringsheim T M, et. al. (2019) Correction: Age specific global epidemiology of hydrocephalus: Systematic review, meta-analysis and global birth surveillance. PloS one 14(1): 0210851.

3. Bhat L, Humphries RL (2021) Neurologic Emergencies. In: Stone C Humphries RL. eds. CURRENT Diagnosis \& Treatment: Emergency Medicine, 8e.

4. Jingjing Lu, Nan Ji, Zhonghua Yang, Xingquan Zhao (2012) Prognosis and treatment of acute hydrocephalus following aneurysmal subarachnoid haemorrhage. J Clin Neurosci 19(5): 669-672.

5. Alselisly A M A, Ashry A H, Mahmoud A T (2021) Hydrocephalus with lateral ventricular lesions: case series and review of literature. Egyptian Journal of Neurology, Psychiatry \& Neurosurgery 57(31): 1-10.

6. Mohammed H, Abd elhakim abd elsatar, Mahmoud Ragab, Ahmed Alghriany (2016) Evaluation of Late Onset Congenital Aqueductal Stenosis Hydrocephalus. J Neurology \& Stroke 5(1): 00163.

7. A Henriette Paulsen, Tryggve Lundar, Karl Fredrik Lindegaard (2010) Twenty year outcome in young adults with childhood hydrocephalus: assessment of surgical outcome, work participation, and health related quality of life. J Neurosurg Pediatr 6(6): 527-535.

8. Stephen M Bergin, Amy L Dunn, Luke G F Smith, Annie I Drapeau (2019) Management of hydrocephalus in infants with severe hemophilia A: report of 2 cases. J Neurosurg Pediatr 23(2): 159-163.

9. Seong Son, Sang Gu Lee, Chan Woo Park, Woo Kyung Kim (2013) Acute Hydrocephalus Following Cervical Spinal Cord Injury. J Korean Neurosurgical Society 54(2):145-147.

10. A Hartmann, E Alberti (1977) Differentiation of communicating hydrocephalus and presenile dementia by continuous recording of cerebrospinal fluid pressure. J Neurol Neurosurg Psychiatry 40(7): 630640 .

11. Mariasanta Napolitano, Sergio Siragusa, Guglielmo Mariani (2017) Factor VII Deficiency: Clinical Phenotype, Genotype and Therapy. J Clin Med 6(4): 38

12. James B Fowler, Orlando De Jesus, Fassil B Mesfin (2021) Ventriculoperitoneal Shunt. Statpearls Publishing, Treasure Island, Florida, USA.

13. Jun Kyeung Ko, Seung Heon Cha, Byung Kwan Choi, Jae Il Lee, Eun Young Yun, et. al. (2014) Hemorrhage Rates Associated with Two Methods of Ventriculostomy: External Ventricular Drainage vs. Ventriculoperitoneal Shunt Procedure. Neurol Med Chi 54(7): 545-551. 